\title{
Effets de la teneur en azote de la ration sur les échanges d'azote ammoniacal et d'urée entre le rumen et le sang chez des chèvres taries
}

\author{
$\mathrm{J}^{\text {Brun-Bellut }}{ }^{1}$, JM Kelly ${ }^{2}$, GW Mathison ${ }^{3}$, \\ RJ Christopherson ${ }^{3}$ \\ IINRA, ENSAIA, 54500 Vandœuvre-lès-Nancy, France; \\ 2 University of Guelph, Ontario, N1G 2W1; \\ 3 University of Alberta, Edmonton, T6G 2P5 Canada
}

\begin{abstract}
Summary - Urea and ammonia exchanges between the rumen and the blood in the "dry" goat : effect of the protein content of the diet. Urea and ammonia exchanges between the rumen and the blood were measured in 4 dry goats which received 2 dietary levels of crude protein (10.0 and $13.3 \% \mathrm{CP}$ ) in a crossover design. With the low CP diet the amount of recycled urea was 5 times higher than with the high CP diet. Ammonia transfer from rumen to blood was higher than recycled nitrogen urea.
\end{abstract}

Les caprins passent pour être des animaux ayant un fort recyclage d'urée dans le rumen. Peu de mesures ont été effectuées sur des chèvres (Obara et Shimbayiashi, 1987, 1988) et celles-ci ne prennent pas en compte les pertes d'ammoniac. Nous avons voulu estimer l'effet de la teneur en matières azotées totales des rations sur les quantités d'ammoniac et d'urée échangées entre le réticulo-rumen et le sang chez des chèvres taries.

Matériel et méthodes - Quatre chèvres Saanen, adultes, taries (PV : $44 \pm 10 \mathrm{~kg}$ ) ont reçu surcessivement 2 rations selon un schéma expérimental d'inversion. Chaque période comportait 3 semaines d'adaptation et 2 semaines de mesure. Les animaux recevaient $1100 \mathrm{~g}$ d'une ration composée de pulpes de betteraves (54\%), de luzerne déshydratée $(20 \%)$, d'orge $(25 \%)$ pour la ration 1 et la même ration complétée avec $0,9 \%$ d'urée pour la ration 2. Après une mesure de digestibilité des rations et des bilans azotés $(7 \mathrm{j})$, les animaux ont reçu une infusion dans le rumen de ${ }^{14} \mathrm{CO}_{3} \mathrm{HNa}(60 \mu \mathrm{Ci}$ par animal) en dose unique. Puis, 12 prélèvements de contenu de rumen sont effectués pendant $24 \mathrm{~h}$ et la teneur en ${ }^{14} \mathrm{C}$ est mesurée sur le $\mathrm{CO}_{2}$ en solution dans chaque échantillon. L'équation de la courbe de diminution de la concentration en ${ }^{14} \mathrm{C}$ permet de déterminer les pertes irréversibles en $\mathrm{CO}_{2}$ du rumen, c'est-à-dire les quantités qui quittent le rumen ou qui sont détruites, par unité de temps. Puis une infusion continue de $\left({ }^{15} \mathrm{NH}_{4}\right)_{2} \mathrm{SO}_{4}$ est réalisée pendant $30 \mathrm{~h}$ dans le rumen $\left(30 \mathrm{mg} / \mathrm{j}\right.$ à $99 \%$ de $\left.{ }^{15} \mathrm{~N}\right)$. Au cours des 3 dernières heures, les concentrations en marqueurs du sang et du rumen ayant atteint un "plateau ", 6 prélèvements effectués dans ces 2 milieux permettent de déterminer la concentration en ${ }^{15} \mathrm{~N}$ de l'ammoniac du rumen et de l'urée du sang et donc d'estimer la part de l'azote de l'urée du sang qui provient de l'ammoniac du rumen. Une semaine après ces injections, $100 \mu \mathrm{Ci}$ de $\left({ }^{14} \mathrm{C}\right)$ urée sont infusés dans le sang pendant $30 \mathrm{~h}(80 \mu \mathrm{Ci} / j)$. Six prélèvements d'échantillons de sang et de contenu de rumen effectués pendant les 3 dernières heures d'infusion permettent d'estimer les pertes irréversibles d'urée et les transferts d'urée du sang au réticulo-rumen (Nolan et Leng, 1972). 
Résultats et discussion - Les quantités d'azote fécal ont été les mêmes avec les 2 rations. En revanche, l'augmentation de l'excrétion urinaire d'azote a représenté $50 \%$ de la quantité d'N supplémentaire ingérée. Les pertes irréversibles d'urée plasmatique (tableau II) peu différentes d'une ration à l'autre, sont plus faibles que celles obtenues par Obara et Shimbayashi (1987). Ces auteurs indiquent des pertes irréversibles comprises entre 16,6 et $35,5 \mathrm{gN} / \mathrm{j}$ pour des urémies de 320 à $700 \mathrm{mg} / \mathrm{l}$ (176 et $225 \mathrm{mg} / \mathrm{l}$ dans notre essai). Quand les chèvres consomment la ration la plus pauvre en azote, elles recyclent des quantités d'urée 5 fois supérieures à celles obtenues avec l'autre ration.

Les quantités d'azote ammoniacal issues du rumen, retrouvées dans l'urée sanguine, représentent 20 et $27 \%$ de l'azote ingéré. Si l'on admet que l'ammoniac provient directement du rumen (Nolan et Leng 1972), le bilan net des échanges d'azote entre le rumen et le sang est négatif, que les animaux reçoivent une ration riche ou pauvre en matières azotées. Les teneurs élevées en azote ammoniacal du contenu de rumen (tableau I) confirment que l'apport d'azote fermentescible est supérieur aux capacités de capture des microorganismes. Ces résultats semblent indiquer que, pour des chèvres recevant des quantités d'aliments limitées, la quantité d'azote mise à la disposition des microorganismes du rumen est sous-estimée. En outre, l'évaluation du recyclage d'azote uréique n'est pas suffisante pour connaître les quantités d'azote mises à la disposition des microorganismes du rumen.

Nolan JV, Leng RA (1972) Br J Nutr 27, 177 Obara Y, Shimbayashi K (1987) Jpn J Zootech Sci 58 (7), 611

Obara Y, Shimbayashi K (1988) Jpn J Zootech Sci 59 (7), 604

Tableau I. Ingestion ef utilisation de l'azote.

\begin{tabular}{ccccccccc}
\hline Ration & $\begin{array}{c}N \text { ingéré } \\
g / j\end{array}$ & $\begin{array}{c}d P D I \\
g / j\end{array}$ & $\begin{array}{c}P D I E \\
g / j\end{array}$ & UFL & $\begin{array}{c}N \text { fèces } \\
g / j\end{array}$ & $\begin{array}{c}N \text { urine } \\
g / j\end{array}$ & $\begin{array}{c}\text { Urémie } \\
m g / l\end{array}$ & $\begin{array}{c}N H_{3} \text { rumen } \\
m g N / I\end{array}$ \\
\hline 1 & 17,22 & -12 & 90 & 0,96 & 5,04 & 7,48 & 176 & 127 \\
2 & 21,75 & 4 & 86 & 1,00 & 5,17 & $\begin{array}{c}9,82 \\
S\end{array}$ & 225 & 174 \\
& $S$ & & & & & & & \\
\hline
\end{tabular}

Tableau II. Échanges entre le rumen et le sang ( $\mathrm{gN} / \mathrm{j}$ ).

\begin{tabular}{|c|c|c|c|c|c|}
\hline \multirow[t]{2}{*}{ Ration } & \multicolumn{2}{|c|}{ Urée } & \multicolumn{2}{|c|}{$\mathrm{NH}_{3}$ rumen } & \multirow{2}{*}{$\begin{array}{c}\text { Azote net } \\
\text { recyclé }\end{array}$} \\
\hline & pertes & recyclé & pertes & trans* & \\
\hline $\begin{array}{l}1 \\
2\end{array}$ & $\begin{array}{l}6,2 \\
7,1\end{array}$ & $\begin{array}{l}1,2 \\
0,2\end{array}$ & $\begin{array}{l}12,3 \\
17,8\end{array}$ & $\begin{array}{l}2,9 \\
5,5\end{array}$ & $\begin{array}{c}-1,7 \\
-5,3 \\
s\end{array}$ \\
\hline
\end{tabular}

- Transfert du rumen au sang. $S$ : significatif : $P<0,05$. 